\title{
Twelve Years of Experience in the Management of Testicular Germ Cell Tumors at a Referral Center in Portugal
}

\author{
Diana Valadares ${ }^{\mathrm{a}, \mathrm{c}}$, Filipe Nery ${ }^{\mathrm{a}}$, Franklim Marques ${ }^{\mathrm{a}, \mathrm{b}}$
}

\begin{abstract}
Background: Testicular germ cell tumors (TGCT) are generally rare but quite frequent in young males. Guidelines are well established for their management.
\end{abstract}

Methods: We present the first report from Portugal on clinical, histological, treatment modalities and outcomes of a population with TGCT. Data was retrospectively analyzed for the 1996 through 2008 period, applying a previous internally validated protocol.

Results: Seventy nine patients with TGCT were identified, $40.5 \%$ had seminomatous and $59.5 \%$ nonseminomatous tumors. Incidence rates were higher among males in their twenties and thirties. Pain and swelling testis were the most common symptoms and microlithiasis was detected in $20.3 \%$ of patients. Lower stages were more frequent in seminomatous tumors. Orchiectomy was done in all patients and further therapy was performed by guidelines recommendations in $86.1 \%$ of them. Hematological toxicity was found in $44.3 \%$ of the population studied and free disease survival rates were at $88.6 \%$.

Conclusions: This retrospective study corroborates the European Western country trends concerning TGCT. Mortality was only seen in nonseminomatous TGCT group. Good risk and lower TGCT stages have no deaths reported. Public health campaigns should be undertaken to guide patients to seek medical advice earlier in the course of the disease.

Manuscript accepted for publication September 30, 2010

${ }^{a}$ Department of Internal Medicine, Centro Hospitalar do Porto Hospital Santo Antonio, Porto, Portugal

${ }^{\mathrm{b}}$ Director of Oncology Services, Centro Hospitalar do Porto - Hospital

Santo Antonio, Porto, Portugal

${ }^{\mathrm{c}}$ Corresponding author: Department of Internal Medicine, Centro Hospitalar do Porto - Hospital Santo Antonio. Largo Prof Abel Salazar 4099-001 Porto, Portugal. Email: divaladares@gmail.com

doi: $10.4021 /$ wjon $245 w$
Keywords: Testicular germ-cell tumors; Risk factors; Incidence; Outcome

\section{Introduction}

Testicular germ cell tumors (TGCT) are a rare malignant disease, counting for 1 to $2 \%$ of all male cancers. However, they are the most common malignancy in the younger generations [1]. In Europe, it seems that incidence has been rising in the past years with decreasing mortality rates. In Portugal, the incidence of TGCT is $2.3 / 100,000$, below the European average of $4.2 / 100,000$, with mortality rates of $0.3 / 100,000$, which seem to be increasing $[2,3]$. TGCT has a very good prognosis and even in metastatic disease the cure rates are near $80 \%$ [4].

There are many known risk factors to develop TGCT (previous contralateral TGCT, family history, cryptorchidism, klinefelter syndrome, etc) [5]. International guidelines are available to facilitate correct diagnosis, treatment and follow-up [6].

The authors intend to present the experience in managing TGCT over 12 years, at the Department of Oncology in Centro Hospitalar do Porto, Hospital Santo Antonio in Portugal. They analyzed risk factors for TGCT, stage at the time of diagnosis, histological characteristics, management, treatment options and survival. To our knowledge this is the first report ever done in Portugal, to assess the country's status and ranking in international setting.

\section{Materials and Methods}

From pathology department cancer database of our hospital, 85 testicular cancers were recorded between 1996 and 2008. A total of 79 cases were identified as being testicular germ cell tumors. From the six cases excluded, two were non-Hodgkin lymphomas, one Leyding-cell tumor, one leiomyosarcoma, one liposarcoma and one sex cord-stromal tumor.

All the patients identified were treated and followed in 


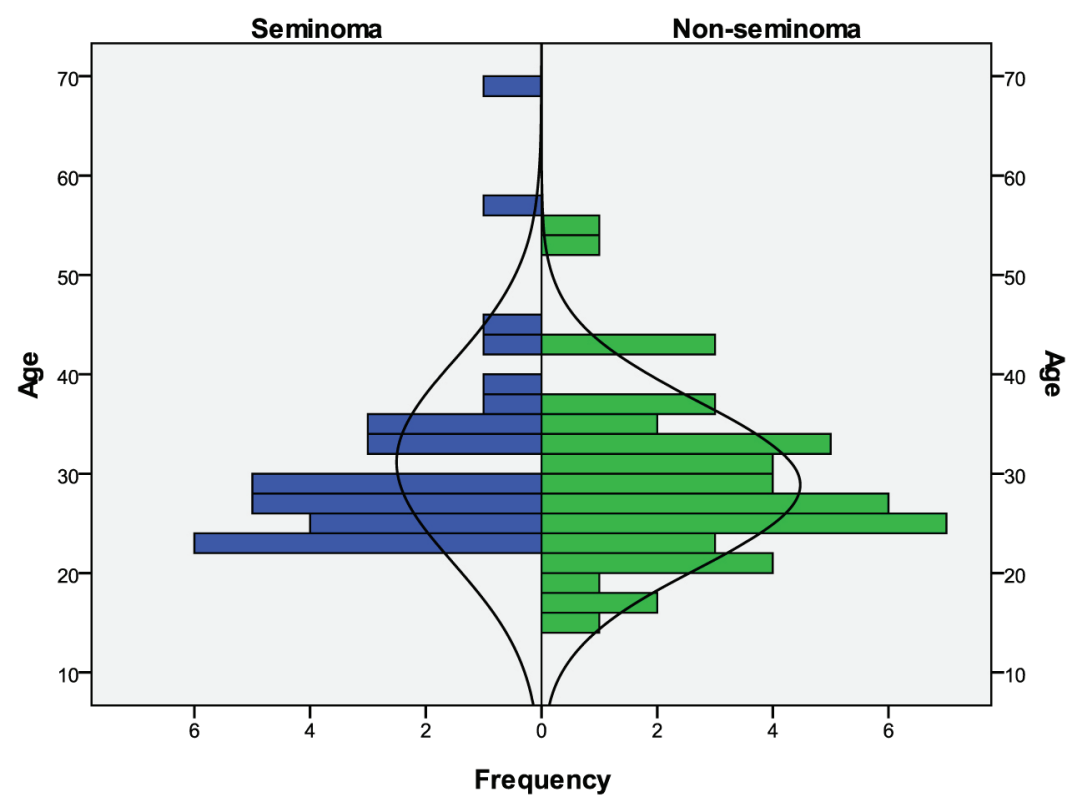

Figure 1. Age distribution considering both subgroups: STCGT and NSTCGT.

our center. All the files were analyzed, applying a protocol covering risk factors, presentation form, initial stage, surgical procedure, histology, concomitant treatment and response, time free of disease, relapse, salvage treatment whenever done, lateral effects, mortality (cancer and non-related mortality). Staging was done using the TNM system created by the American Joint Committee on Cancer (AJCC). Tissue specimens were reviewed in our center and World Health
Organization (WHO) classification for testicular cancer was applied. Patients were broadly divided in two subgroups, i.e., seminomas (STGCT) and nonseminomas (NSTGCT).

All the patients remained under surveillance in our center and latest medical visit of each one was recorded in current year (2010) to extrapolate survival.

Statistical data were computed and analyzed using SPSS version 17.0 software. Descriptive statistics were used to

Table 1. Clinical Symptoms at Diagnosis in STGCT and NSTGCT

\begin{tabular}{llll}
\hline & Symptoms & STGCT (\%) & NSTGCT (\%) \\
\hline \multirow{2}{*}{ Localized } & Testicular atrophy & 0 & $1(2.1 \%)$ \\
& Swelling testis & $6(18.7 \%)$ & $9(19.1 \%)$ \\
& Pain & $9(28.1 \%)$ & $14(29.8 \%)$ \\
& Post traumatic pain & 0 & $1(2.1 \%)$ \\
Disseminated & Breast enlargement & 0 & $4(8.5 \%)$ \\
& Right atrium thrombus & 0 & $1(2.1 \%)$ \\
& Gastro-intestinal & 0 & $2(4.3 \%)$ \\
& Retroperitoneal ganglionar & $1(3.1 \%)$ & $8(17.0 \%)$ \\
& Lumbar pain & $2(6.2 \%)$ & $5(10.6 \%)$ \\
& Bone pain & $1(3.1 \%)$ & $2(4.3 \%)$ \\
& Central nervous system & $1(3.1 \%)$ & 0 \\
& Leg swelling & 0 & $1(2.1 \%)$ \\
\hline
\end{tabular}


Table 2. Histological Characteristics and Different Stages of Both TGCT Groups

\begin{tabular}{|c|c|c|c|c|c|c|c|c|c|c|}
\hline \multirow[t]{2}{*}{ Type } & \multirow{2}{*}{$\begin{array}{l}\text { No. of } \\
\text { cases }\end{array}$} & \multicolumn{9}{|c|}{ Stage } \\
\hline & & IA & IB & IS & IIA & IIB & IIC & IIIA & IIIB & IIIC \\
\hline Seminomas & 32 & 11 & 3 & 10 & 2 & 3 & 0 & 0 & 2 & 1 \\
\hline Nonseminomas & 47 & 3 & 2 & 13 & 4 & 6 & 1 & 0 & 10 & 8 \\
\hline Embryonal Carcinoma & 14 & 2 & 1 & 2 & 2 & 1 & 0 & 0 & 3 & 3 \\
\hline Mature Teratoma & 1 & 0 & 0 & 1 & 0 & 0 & 0 & 0 & 0 & 0 \\
\hline Imature Teratoma & 1 & 0 & 0 & 0 & 0 & 0 & 0 & 0 & 1 & 0 \\
\hline Choriocarcinoma & 2 & 0 & 0 & 0 & 0 & 0 & 0 & 0 & 1 & 1 \\
\hline Mixed tumors & 28 & 1 & 1 & 10 & 1 & 5 & 1 & 0 & 5 & 4 \\
\hline Teratocarcinoma & 1 & 0 & 0 & 0 & 1 & 0 & 0 & 0 & 0 & 0 \\
\hline
\end{tabular}

characterize the most relevant clinical parameters. Actuarial survival rates were estimated using the Kaplan-Meier product limit method, and differences observed among patient subgroups were assessed by the log-rank test.

\section{Results}

A total of 79 male patients were identified as having TGCT. From those, 32 (40.5\%) were STCGT and 47 (59.5\%) NSTGCT. Overall mean age at diagnosis was 29.8 years old, with the youngest boy being 15 years old, and the oldest patient being 69 years old. Mean age at diagnosis for seminomas was 31.2 and 28.9 years old for nonseminomas, peaking in their twenties and thirties (Fig 1). Two patients were HIV and six HCV positive.

Microlithiasis occurred in $20.3 \%$ of the patients. Cryptorchidism, infertility and previously testicular surgery each counted for 3.8\%. Previous testicular cancer, testicular atrophy and varicocele were present in $2.5 \%$.

The most common symptoms at presentation were tes- ticular pain and swelling testis in identical percentages in both groups. Symptomatic metastasis was more frequently found in nonseminomatous TGCT (Table 1).

Referral to the CHP was mostly done by general practitioners $(59.5 \%)$, followed by patients who sought medical attention at the emergency department due to some of the symptoms mentioned above (35.4\%) and finally $5.1 \%$ of the patients were sent by physicians of neighboring hospitals.

All of the patients underwent testicular ultrasound, thoraco-abdomino-pelvian $\mathrm{CT}$ scan and tumoral markers $(\alpha$-fetoprotein (AFP), $\beta$-human chorionic gonadotrophin $(\beta-h C G)$ and lactic dehydrogenase (LDH), before surgical procedure.

After staging, NSTGCT were more frequently found to have disseminated disease, as expressed by $57.4 \%$ of the patients opposed to $25 \%$ in the seminomatous group.

As expressed in Table 2, patients with STGCT were diagnosed with lower stages. A more heterogeneous distribution was found among nonseminomatous group. In this last group mixed tumors and embryonal carcinoma tumors were more prevalent.

Table 3. Risk Classification

Risk Classification

\begin{tabular}{lccccc}
\hline & \multicolumn{2}{c}{ Good } & Intermediate & Poor \\
\cline { 2 - 6 } & STGCT & NSTGCT & STGCT & NSTCGT & NSTGCT \\
No residual disease & 29 & 23 & 1 & 4 & 1 \\
Residual disease & 0 & 5 & 1 & 5 & 5 \\
Progression & 1 & 1 & 0 & 2 & 1 \\
\hline
\end{tabular}


Table 4. Global Treatment Modalities Applied in Different Stages of TGCT.

\begin{tabular}{ccccccc}
\hline Stage & \multicolumn{3}{c}{ Seminomatous } & \multicolumn{3}{c}{ Nonseminomatous } \\
\hline & CT & RT & $\begin{array}{c}\text { Total of } \\
\text { patients }\end{array}$ & CT & RT & $\begin{array}{c}\text { Total of } \\
\text { patients }\end{array}$ \\
IA & 1 & 5 & 11 & 1 & 1 & 3 \\
IB & 3 & - & 3 & 2 & - & 2 \\
IS & 8 & 2 & 10 & 8 & 1 & 13 \\
IIA & 2 & - & 2 & 3 & - & 4 \\
IIB & 3 & - & 3 & 6 & - & 6 \\
IIC & - & - & - & 1 & - & 1 \\
IIIB & 2 & - & 2 & 10 & - & 10 \\
IIIC & 1 & - & 1 & 8 & - & 8 \\
\hline
\end{tabular}

CT: Chemotherapy; RT: Radiotherapy

Risk classification and prognosis are listed in Table 3, conferring better prognosis among the seminomatous group.

All the patients in both TGCT groups underwent radical orchiectomy. Complementary therapy is as described in Table 4. Radiotherapy was done in lower stages of the disease and chemotherapy was done in all the patients in stages II and III but one in stage IIA in the group of nonseminomatous (this patient was temporarily lost after surgery by CHP).

After the initial approach (surveillance or complemen- tary therapy), a re-evaluation was done. In the seminomatous group, only one patient with a previous stage IA progressed and another one with IIIB stage had residual disease. In the nonseminomatous group, one patient with IIA stage progressed (cited above). The same happened to two patients of IIIB and one IIIC stages. In the nonseminomatous group, four patients with IIB, one patient IIC, five IIIB and another five patients with IIIC stages had residual disease.

Intermediate and poor risk patients with nonseminoma-

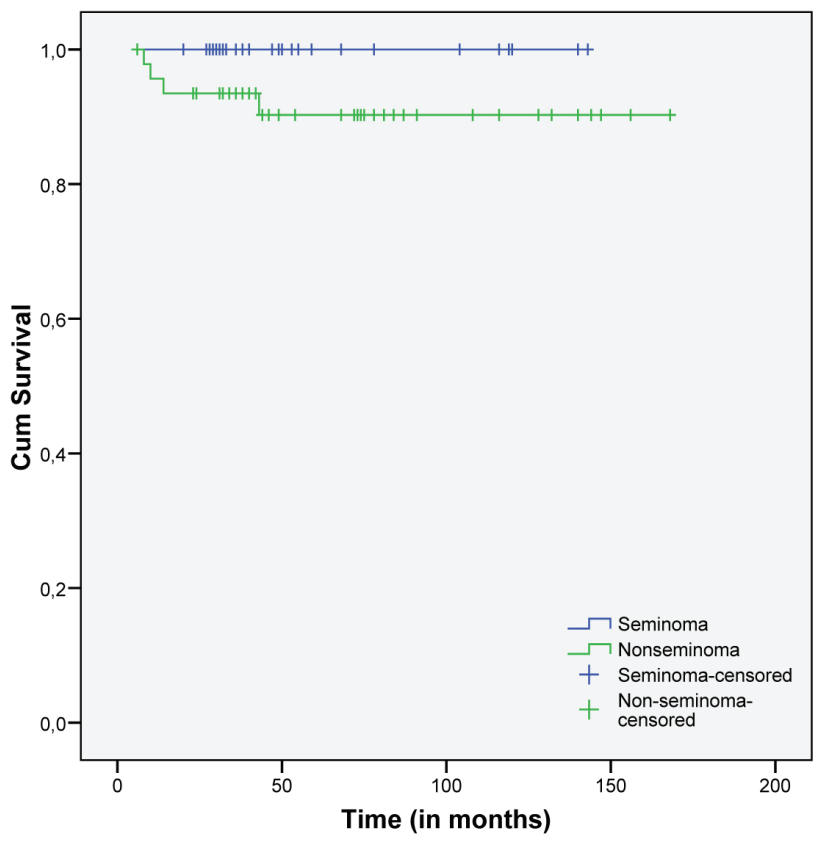

Figure 2. Cumulative survival in both subgroups of TGCT. 


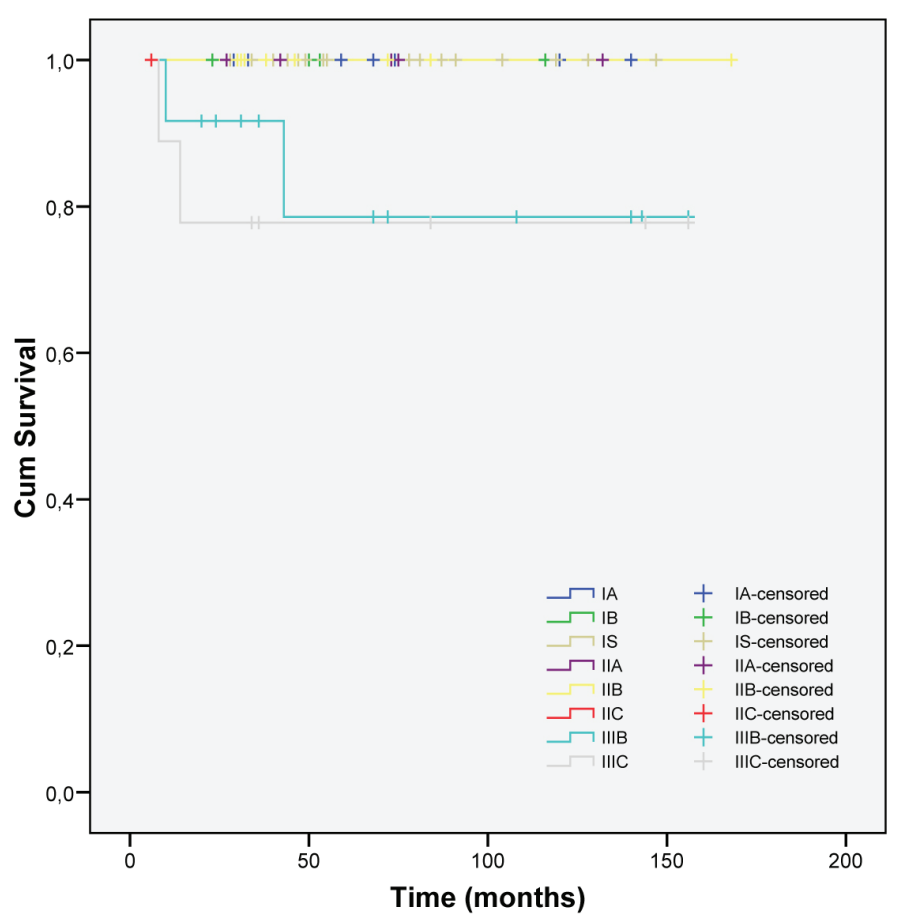

Figure 3. Cumulative survival in all initial stages.

tous TGCT had a tendency to have residual or progression of the disease, as demonstrated by $63.3 \%$ (7/11 patients) and $85.7 \%$ (6/7 patients) respectively (Table 3 ).

After re-evaluation, residual disease was found in 16 $(20.25 \%)$ patients in both groups, all of them presenting retroperitoneal conglomerate. Ten of them underwent retroperitoneal lymph node dissection (RPLND). One received additional chemotherapy (CT) and RPLND, another two just $\mathrm{CT}$, one radiotherapy (RT) and two of them were kept under a surveillance program without any other treatment. It is of notice that from the 16 patients with residual disease, 15 had NSTGCT.

Fifty-eight patients after re-evaluation were considered free of disease, 30 of them (93.75\%) had STGCT and 28 $(59.6 \%)$ had NSTGCT. Four of them had a recurrence after an average of 19.25 months (minimum of 10 and maximum 39 months). Two were seminomas (stages IS and IA) and the other ones nonseminomas (IS and IIIC). Retroperitoneum and liver were the organs where metastasis was found.

Adverse effects attributed to medical treatment were found in 30 patients. Hematologic toxicity was the most prevailing one at $44.3 \%$ ( 27 out of 61 patients that received chemotherapy), lung at $4.08 \%$, dermatologic at $3.3 \%$ and raynaud phenomena in 3.3\%.

At the end of the study $89.9 \%$ of the patients were still alive. Eight patients died, four of them with NSTGCT. Death was attributed to cancer, two not related to TGCT and the other two related to a complication of the treatment.
In almost $1 / 3$ of all patients $(27.8 \%)$ a testicular prosthesis was implemented and semen cryopreservation was done in $30.4 \%$.

Overall survival was lower in NSTGCT but with no statistical significance $(\mathrm{p}=0.102)$, although all patients with STGCT were still alive (Fig. 2).

More advanced stages were related to a low survival (intermediate and poor risk) (Fig. 3 and 4).

\section{Discussion}

To our knowledge, this is the first report ever made in Portugal about clinical features, treatment and outcome in testicular tumors.

TGCT has been more frequently diagnosed on males in their twenties and thirties, mirroring the Western Europe incidence, with no significant differences between the two histological types [2].

Testicular microlithiasis may be a risk factor for the development of TGCT, but the association between this finding and testicular cancer is highly variable depending on the studies published [7]. A prospective study found only one $(1.6 \%)$ out of 63 patients with microlithiasis as having developed a NSTGCT, and it concluded that self-examination should be done instead of an intensive screening imagery surveillance program [8]. In our population with TGCT we found $20.3 \%$ of patients with testicular microlithiasis. Its 


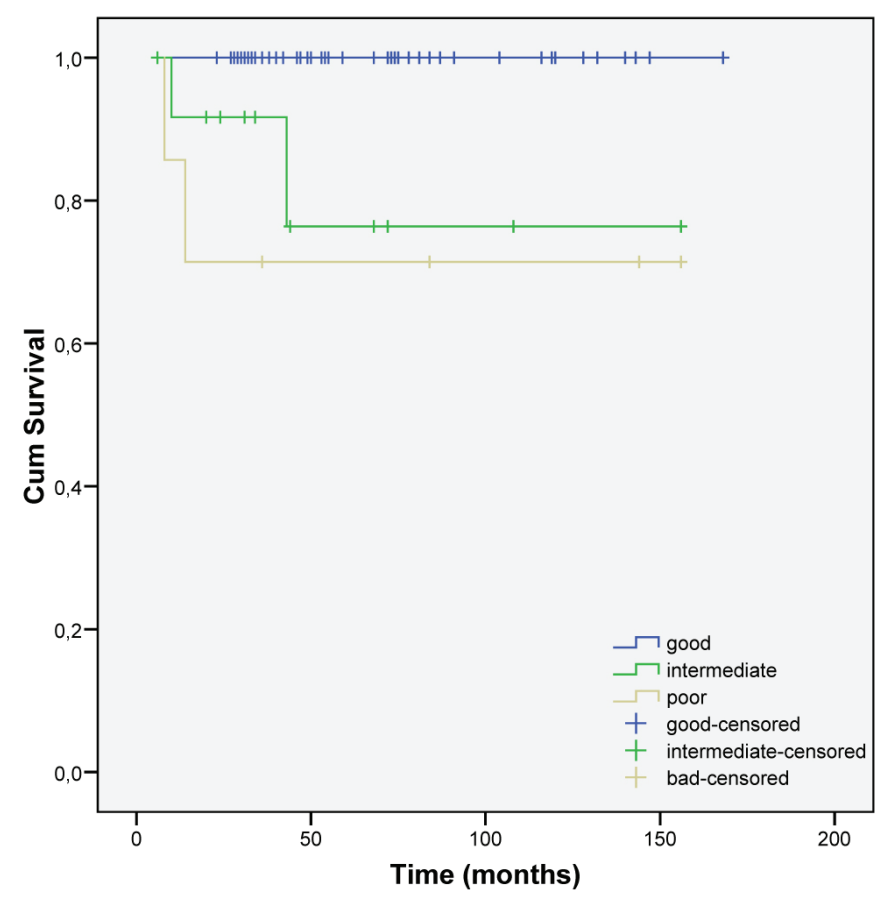

Figure 4. Cumulative survival according to risk score stratification.

meaning as a risk factor for the development of TGCT may be questionable. As we don't have the prevalence of testicular microlithiasis in the Portuguese healthy male population, this finding on $1 / 5$ of the patients with TGCT should be interpreted as frequent association with TGCT.

Six patients (7. 6\%) were found to be HCV and two HIV positive. It is known that HIV increases the incidence of TGCT, but there are no studies concerning the implication of $\mathrm{HCV}$ in testicular tumorogenesis [9]. Metachronous tumors were found in two patients, with the second tumor developing more than five years after the first one, as described elsewhere. This leads to a recommendation for long-term monitoring [10].

The most frequent clinical features at diagnosis were the localized ones such as pain and swelling testis, with similar prevalences among both groups. It was in the nonseminomatous group that symptoms of disseminated disease were most frequent. They presented a more severe features and a worse risk stratification that correlates with poor survival.

In spite of its after-the-fact approach, it was possible to access to all imagery and biochemical exams (testicular echography and CT scans) done at the time of diagnosis and before the surgical procedures, and it does demonstrate good compliance with the institution protocol.

The finding of a more severe disease by the time of presentation revealed by the patients symptoms in the nonseminomatous subgroup was confirmed after the initial staging. This was demonstrated by more than doubling of the dis- seminated disease in this group and a worse outcome.

STGCT presented with lower stages of disease and nonseminomatous tumors have a wider distribution. Higher stages are found more frequently in this subgroup.

Therapeutic options for treatment of TGCT patients have not changed much over recent years. This retrospective review of 12 years of experience also demonstrated this reality.

In the early stages (clinical stage I) of STGCT radiotherapy, chemotherapy or surveillance after initial diagnostic and therapeutic orchiectomy are all options to be considered [11]. Of the 24 patients in stage I, seven of them underwent adjuvant radiotherapy (20 to $30 \mathrm{~Gy}$ ), ten received single carboplatin cycle, one BEPx3, one two cycles of carboplatin and five patients got only regular surveillance. None of the patients that were submitted to RT or carboplatin single cycle have relapsed or died, with mean surveillance follow-up of $68.0 \pm 34.70$ and $40.0 \pm 14.70$ months respectively. Both patients that were submitted to more aggressive chemotherapeutic schemes had vascular or spermatic cord invasion. One of them (stage IS) relapsed after 59 months, with visceral metastasis. A salvage treatment was done with BEP, but the patient died one month after metastatic disease was recognized. This was related to chemotherapy complications.

Nowadays, RT is not an option for early NSTGCT, but adjuvant chemotherapy or even tight surveillance may be [12]. From the 18 patients in this subgroup, six underwent BEPx3, four BEPx2, one received single carboplatin cycle 
and five were only subject to surgical procedure. Two patients received radiotherapy after orchiectomy in the late 1990 `s, and both were still alive. In those days this RT treatment type was acceptable. Whatever type of treatment provided, the clinical response was similar. Cure rates were at $100 \%$.

In 34 patients with retroperitoneal lymph node metastasis at presentation, confirming more advanced clinical stages, and after initial treatment (orchiectomy, chemotherapy, and orchiectomy with concomitant RPLND in only one patient), re-evaluation showed 14 were free of disease and sixteen with residual disease. After the initial chemotherapy 11 patients with residual disease underwent RPLND, as recommended. Two (18.2\%) of them died but none of the deaths was related to disease progression. So, all the patients that underwent RPLND had their malignancy controlled, without relapse, conferring better prognosis than what is described elsewhere (risk of relapse in similar patients from 12 to $45 \%$ ) [13].

Adverse side effects attributed to medical treatment are probably underestimated. The most frequently found were hematologic toxicity in $43.3 \%$, and pulmonary toxicity in $4.08 \%$ of patients, attributed to bleomycin (49 patients received chemotherapy schemes with bleomycin sometime over the course of the disease). This is in line with what is described in literature [14].

Disease free survival rate is globally estimated to be $88.6 \%$, with a median time of surveillance of $46 \pm 34.7$ months. Death attributed to testicular cancer (5.06\%) happened only in the nonseminomatous group, and in patients with advanced initial stages (IIIB and IIIC). Prognosis of our patients was clearly influenced by the initial stage, as expected [15].

The promptness of the starting of treatment (as measured by a median of 8 days (IQR $=4-21.5$ ) from the referral of patients until the start of treatment ) may have impacted the good outcome. Unfortunately, seeking a doctor's help at the beginning of the symptoms is not so prompt, with a median of 21 days $(\mathrm{IQR}=3-60)$, and that is probably an underestimation.

We don't know if HCV infection has any role in tumorigenesis of TGCT. It could be just a simple coincidence, but it may be a future direction for other studies.

NSTGCT presented with worse prognosis, more advanced disease at presentation and survival rates that are lower than the STGCT subgroup. This later one had an excellent outcome independent of the stage or risk group stratification.

In conclusion, public health campaigns should be carried out to alert and teach how to make a testicular self exam to reduce the time from the beginning of symptoms and the time of seeking expert help. This may well improve the outcomes and cure rates.

\section{References}

1. Huyghe E, Matsuda T, Thonneau P. Increasing incidence of testicular cancer worldwide: a review. J Urol 2003;170(1):5-11.

2. Huyghe E, Plante P, Thonneau PF. Testicular cancer variations in time and space in Europe. Eur Urol 2007;51(3):621-628.

3. www.globocan.iarc.fr

4. Feldman DR, Motzer RJ. Good-risk-advanced germ cell tumors: historical perspective and current standards of care. World J Urol 2009;27(4):463-470.

5. Parker L. Causes of testicular cancer. Lancet 1997;350(9081):827-828.

6. Motzer RJ, Agarwal N, Beard C, Bolger GB, Boston B, Carducci MA, Choueiri TK, et al. NCCN clinical practice guidelines in oncology: testicular cancer. J Natl Compr Canc Netw 2009;7(6):672-693.

7. Rashid HH, Cos LR, Weinberg E, Messing EM. Testicular microlithiasis: a review and its association with testicular cancer. Urol Oncol 2004;22(4):285-289.

8. DeCastro BJ, Peterson AC, Costabile RA. A 5-year followup study of asymptomatic men with testicular microlithiasis. J Urol 2008;179(4):1420-1423; discussion 1423.

9. Milosevic MF, Gospodarowicz M, Warde P. Management of testicular seminoma. Semin Surg Oncol 1999; 17(4):240-249.

10. Nery F, Valadares D, Marques F. Metachronous Testicular Germ-cell Tumors: The Importance of a Long-Term Follow-up. World J Oncol 2010; 1(3):145-7

11. Chung P, Mayhew LA, Warde P, Winquist E, Lukka H. Management of stage I seminomatous testicular cancer: a systematic review. Clin Oncol (R Coll Radiol) 2010;22(1):6-16.

12. Hotte SJ, Mayhew LA, Jewett M, Chin J, Winquist E. Management of stage I non-seminomatous testicular cancer: a systematic review and meta-analysis. Clin Oncol (R Coll Radiol) 2010;22(1):17-26.

13. Culine S. Editorial comment on: postchemotherapy retroperitoneal lymph node dissection in advanced germ cell cancer tumors of the testis. Eur Urol 2008;53(2):273274.

14. Simpson AB, Paul J, Graham J, Kaye SB. Fatal bleomycin pulmonary toxicity in the west of Scotland 1991-95: a review of patients with germ cell tumours. Br J Cancer 1998;78(8):1061-1066.

15. Howard GC, Conkey DS, Peoples S, McLaren DB, Hargreave TB, Tulloch DN, Walker W, et al. The management and outcome of patients with germ-cell tumours treated in the Edinburgh Cancer Centre between 1988 and 2002. Clin Oncol (R Coll Radiol) 2005;17(6):435440 . 